\title{
Comparison of UHPLC-ESI-MS and Hadamard Transform Atmospheric Pressure Ion Mobility-ESI-MS for Rapid Profiling of Isomeric Flavonoids
}

\author{
Michael Groessla ${ }^{\text {, Antonio Azzollinib, Philippe J. Eugster }}{ }^{\mathrm{b}}$, Benoit Plet ${ }^{\mathrm{a}}$, Jean-Luc Wolfender ${ }^{\mathrm{b}}$, \\ and Richard Knochenmuss ${ }^{\star a}$
}

\begin{abstract}
Hadamard transform atmospheric pressure ion mobility-MS and rapid UHPLC-MS methods were investigated for analysis of closely related isomeric flavonoids and their glycosides using a test set of seven standards. On a time scale of a few minutes, the flavonoid aglycones were all separated by ion mobility, but not by UHPLC. The glycosides were better resolved by IMS but not completely separated by both methods. The results suggest that IMS provides sufficient resolution for separation of isomeric polyphenols such as flavonoids in high-throughput metabolomics studies.
\end{abstract}

Keywords: ESI-TOF-MS · Flavonoids · Glycosides · Ion mobility · UHPLC

\section{Introduction}

The analysis of natural products in complex extracts represents a major analytical challenge because of their high chemodiversity and the size of plant metabolomes. For efficient profiling high efficiency separations, high sensitivity and high dynamic range are needed. Among the most widely applied methods, high-performance liquid chromatography (HPLC) or ultra-high pressure liquid chromatography (UHPLC) coupled with mass spectrometry (MS) have been successfully applied to the investigation of complex plant extracts. ${ }^{[1]}$ Direct infusion methods taking advantage of ultra-high resolution MS instruments have also been used to profile extracts without prior LC separation. Both approaches are extensively used for plant metabolomics. ${ }^{[2]}$

Natural extracts contain a wide range of secondary metabolites, and for many of them multiple isomers may be found in a single metabolome. This is particularly true for flavonoids, a class of polyphenols

\footnotetext{
${ }^{*}$ Correspondence: Dr. R. Knochenmuss ${ }^{a}$ Tel.: +41335111167

E-mail: knochenmuss@tofwerk.com aTofwerk AG

Uttigenstrasse 22

$\mathrm{CH}-3600$ Thun

'School of Pharmaceutical Sciences, EPGL University of Geneva, University of Lausanne 30 Quai Ernest-Ansermet

$\mathrm{CH}-1211$ Geneva 4
}

ubiquitous in flowering plants. Flavonoids differ in their oxidation pattern and subtypes, and also occur as glycosides that are substituted on various positions of the polyphenol aglycone. ${ }^{[3]}$

This multiplicity of isomers significantly increases the difficulty of the separation step prior to mass spectrometry. Typically, separation of flavonoids by LC requires careful optimization, and run times may become undesirably long. ${ }^{[3]}$ Obviously, isomers can never be resolved with a direct infusion method, regardless of the resolving power of a single stage mass analyser (no MS/MS).

In such cases ion mobility spectrometry (IMS) can add a complementary separation dimension without increasing cycle time, and with minimal added experimental complexity. IMS separates on the millisecond time scale, ${ }^{[4]}$ which lies between the minutes of LC separation and the microseconds of (time-of-flight, TOF) mass spectrometry. IMS also has the advantage that reduced mobilities and collision cross sections of ions are physical quantities which are calculable, reproducible and transferable between instruments. In contrast, retention times in LC have stability and reproducibility issues.

While atmospheric pressure IMS-MS has been applied to complex samples, ${ }^{[5]}$ it has suffered from relatively low sensitivity imposed by the 'pulse-and-wait' mode of operation of drift tube instruments. A packet of ions is gated into the drift tube, after which the gate is closed until the slowest ions have reached the end of the drift tube. The corresponding duty cycle is usually below $1 \%$, meaning a pulsed IMSMS instrument loses at least 2 orders of sensitivity compared to MS alone.

The duty cycle can be raised by various methods, including by Hadamard modulation of the ion current. ${ }^{[6]}$ The Hadamard transform (HT) has been applied to IMS ${ }^{[7-9]}$ but has not yet achieved wide acceptance, in spite of its $50 \%$ duty cycle. One reason for this is that numerous effects, such as modulation imperfections, can seriously degrade the quality of the transformed spectrum. These and other artefacts have been successfully eliminated or suppressed in a new generation of HT-IMS-MS spectrometers ${ }^{[10]}$ and applied to complex samples such as human plasma.[11]

In this paper, the HT-IMS-TOF instrument is compared with UHPLC-MS for the rapid resolution of isomeric flavonoids and their glycosides. The UHPLC-MS was operated with fast, high throughput generic linear gradients (4 min) that are typically used for metabolomics. The relative capabilities and limits of both methods are investigated in terms of resolution power for artificial mixture of isomeric flavonoids that are likely to occur in plant extracts.

The test compounds are shown in Fig. 1. While closely related, the flavonoid aglycones may be expected to have relatively well-defined structures as gas-phase ions, with few low energy, easily interconverting conformations. This is favourable for IMS separation, but does not guarantee that the mobilities will be measurably different. The glycosides are a potentially more difficult set, with more thermally ac- 
<smiles>O=c1cc(-c2ccc(O)c(O)c2)oc2cc(O)cc(O)c12</smiles>

Luteolin<smiles>C[C@H](OC(C(C)(C)C)C(C)(C)C)C(C)(C)C</smiles>

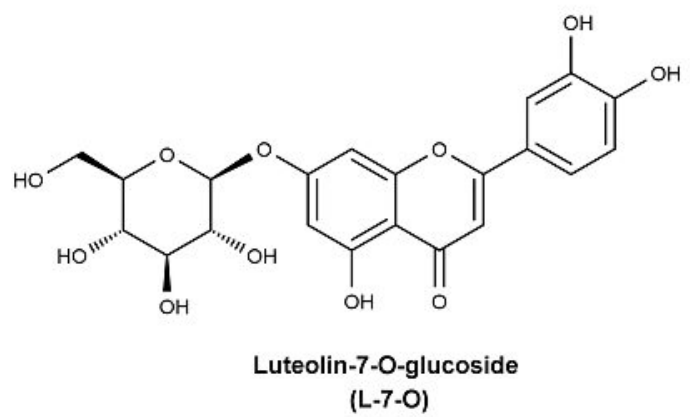<smiles>O=c1c(O)c(-c2ccccc2O)oc2cc(O)cc(O)c12</smiles>

Datiscetin<smiles>O=c1c(O)c(-c2ccc(O)cc2)oc2cc(O)cc(O)c12</smiles>

Kaempferol<smiles>O=c1c(O[C@@H]2O[C@H](CO)[C@@H](O)[C@H](O)[C@H]2O)c(-c2ccc(O)cc2)oc2cc(O)cc(O)c12</smiles>

Kaempferol-3-O-glucoside (K-3-O)<smiles>O=c1c(O)c(-c2ccc(O)cc2)oc2cc(O[C@@H]3O[C@H](CO)[C@@H](O)[C@H](O)[C@H]3O)cc(O)c12</smiles>

Kaempferol-7-O-glucoside (K-7-O)

Fig. 1. Structures of the compounds included in this study.

cessible internal degrees of freedom. This is less favourable for IMS, since interconversion between conformations will broaden the mobility peak, reducing separation.

\section{Experimental}

\section{Chemicals}

All flavonoids and glycosides were obtained from Extrasynthese (Genay, France) and used without further purification. For IMS-MS, LC-MS grade methanol, ethanol, acetonitrile and formic acid (FA) were obtained from Sigma-Aldrich (Buchs, Switzerland). Stock solutions of all compounds $(0.5 \mathrm{mM})$ were prepared in ethanol and diluted with either methanol or acetonitrile, each containing $0.1 \%$ formic acid, to a final concentration of $5 \mu \mathrm{M}$ for IMS-MS measurements in negative ion (NI) mode and $25 \mu \mathrm{M}$ for IMS-MS measurements in positive ionisation (PI) mode. For complexation experiments, sodium chloride (Sigma-Aldrich) was added in twofold molar excess compared to the analyte.

UHPLC-TOF-MS analyses were performed using ULC/MS grade acetonitrile, water and formic acid (FA) obtained from Biosolve (Valkenswaard, The Netherlands). Two samples, one containing the glycosides and the other the aglycones, were prepared at $30 \mu \mathrm{g} / \mathrm{mL}$ in ULC/ MS grade methanol.

\section{Ion Mobility TOF Mass Spectrometry}

All IMS-MS measurements were carried out on a Tofwerk (Thun, Switzerland) Hadamard transform IMS-TOF-MS. The system comprises an ESI source, a $10 \mathrm{~cm}$ desolvation tube, a $20.5 \mathrm{~cm}$ drift tube (both made from resistive glass) and a Tofwerk HTOF TOF-MS. With Hadamard multiplexing, the duty cycle remains at $50 \%$ regardless of resolution or drift time range. Measurements were carried out in both PI and NI mode at an applied ESI potential of $2 \mathrm{kV}$ and $1.7 \mathrm{kV}$, respectively. The desolvation and drift tubes were at ambient atmospheric pressure and $150{ }^{\circ} \mathrm{C}$, with high purity nitrogen as the drift gas. Ion mobility field strengths were $378 \mathrm{~V} / \mathrm{cm}$ (PI mode) and $373 \mathrm{~V} / \mathrm{cm}$ (NI mode). The instrument was calibrated daily, resulting in mass accuracies of $<10 \mathrm{ppm}$. Samples were infused either with a syringe or LC pump at a rate of $1.5 \mu \mathrm{L} / \mathrm{min}$. The total acquisition time was 4 min per sample, equal to the UHPLC separation time. Post-processing of the raw data was performed with IMS Viewer 1.8 (Tofwerk) using IGOR Pro 6.32 (Wavemetrics, USA). This included mobility $\mathrm{S} / \mathrm{N}$ and resolution enhancement. ${ }^{[10]}$ Single ion mobility chromatograms were generated by integrating across the FWHM of the respective mass peaks.

For the determination of reduced mobilities and collision cross sections, a mixture of quaternary alkyl ammonium salts and 2,6-di-tert-butylpyridine (Sigma Aldrich) was used for calibration. ${ }^{[12-14]}$

Reduced mobility constants $\mathrm{K}_{0}$ were calculated using

$$
K_{0}=\frac{L^{2}}{V t_{d}} \cdot \frac{P}{1013.25} \cdot \frac{273.16}{T}
$$

where $\mathrm{L}$ is the length of the drift tube, $\mathrm{V}$ is the applied potential, $t_{d}$ the drift time of the analyte, $\mathrm{P}$ and $\mathrm{T}$ the pressure and temperature in the mobility cell. 
Collision cross sections, $\Omega$, were calculated with the following formula:

$$
\Omega=\frac{V t_{d}}{L^{2}} \frac{3 e z}{16 N}\left(\frac{2 \pi}{k c T}\right)^{1 / 2}\left(\frac{m+M}{m M}\right)^{1 / 2}
$$

where e is the electron charge, $z$ the charge state of the ion, $\mathrm{N}$ the neutral gas number density, $\mathrm{k}$ the Boltzmann constant, $\mathrm{c}$ a correction factor for the actual temperature in the drift tube, $\mathrm{m}$ and $\mathrm{M}$ the molecular masses of the buffer gas and the analyte, respectively.

\section{UHPLC-TOF-Mass Spectrometry}

UHPLC-TOF-MS analyses were performed on a Waters Acquity UPLC system coupled to a Waters MicromassLCT Premier Time-of-Flight mass spectrometer, equipped with an ESI interface. Separations were carried out using a Waters Acquity BEH C18 UPLC column $(50 \times 1 \mathrm{~mm}$ i.d., $1.7 \mu \mathrm{m})$ in gradient mode at a flow rate of $0.3 \mathrm{~mL} / \mathrm{min}$ with the following solvents: (A) $0.1 \%$ FA in water and (B) $0.1 \% \mathrm{FA}$ in acetonitrile. The generic linear gradient was $5 \%$ to $95 \% \mathrm{~B}$ in $4 \mathrm{~min}$, equal to the IMS measurement time. The column was then washed for $0.8 \mathrm{~min}$ with $95 \% \mathrm{~B}$, reconditioned to $5 \% \mathrm{~B}$ in $0.1 \mathrm{~min}$ and finally equilibrated with $5 \% \mathrm{~B}$ in 1.1 min, for a total cycle time of $6 \mathrm{~min}$. The column temperature was maintained at $40{ }^{\circ} \mathrm{C}$. Analyses of each sample $(1.0 \mu \mathrm{L}$

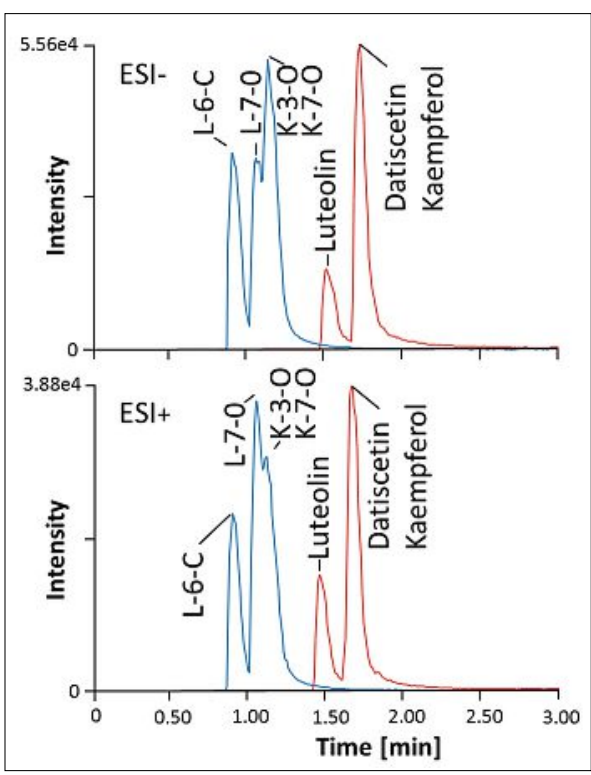

Fig. 2. UHPLC-TOF-MS separation of isomeric flavonoid aglycones (right) and of isomeric flavonoid glycosides (left) in 4 minutes using a $50 \times 1.0 \mathrm{~mm}$ i.d., $1.7 \mu \mathrm{m}$ column in $\mathrm{NI}$ (upper) and PI mode (lower). In both modes, luteolin was baseline separated from the coeluting datiscetin and kaempferol, while L-6-C was baseline separated from the coeluting $\mathrm{K}-3-\mathrm{O}$, $\mathrm{K}-7-\mathrm{O}$ and L-7-O. Intensities were similar in both modes. injected in the partial loop with needle overfill mode) were separately performed in both PI and NI modes in the 100-1000 Da range with acquisition times of $0.25 \mathrm{~s}$ in the centroid mode and an inter-scan delay of $0.01 \mathrm{~s}$. The ESI conditions were set as follows: capillary voltage $2800 \mathrm{~V}$, cone voltage $40 \mathrm{~V}$, source temperature $120{ }^{\circ} \mathrm{C}$, desolvation temperature $250{ }^{\circ} \mathrm{C}$, cone-gas flow $20 \mathrm{~L} / \mathrm{h}$, desolvation gas flow $600 \mathrm{~L} / \mathrm{h}$ and MCP (microchannel plate) detector voltage $2450 \mathrm{~V}$. The MassLynx software 4.1, SCN 639 (Waters, Milford, USA) was used to process the chromatograms. For internal calibration, a $5 \mu \mathrm{g} / \mathrm{mL}$ solution of leucine-enkephalin from Sigma-Aldrich was infused through the lock-mass probe at a flow rate of $5 \mu \mathrm{L} / \mathrm{min}$ using a secondary Shimadzu LC-10ADvp LC pump.

\section{Results and Discussion}

\section{UHPLC-TOF-MS}

Both mixtures of isomeric flavonoid aglycones and glycosides were analysed using a generic fast fingerprinting gradient. Such types of short gradient are typically used in metabolomics studies to generate high number of replicates in a short time, of a large series of samples. Recently, extremely short separations (less than $1 \mathrm{~min}$ ) of crude plant extracts have been shown to be feasible with satisfactory resolution of the main constituents. ${ }^{[1,15]}$ The 4-min generic UHPLC gradient method used here corresponds theoretically to a $83 \mathrm{~min}$ HPLC gradient method, using a $150 \times 4.6$ $\mathrm{mm}$ i.d., $5 \mu \mathrm{m}$ HPLC column, at a flow rate of $1 \mathrm{~mL} / \mathrm{min}$ if the relationship between efficiency and peak capacity is assumed to be strictly linear.[16]

With such UHPLC conditions, however, the isomers were only partly resolved, as shown in Fig. 2. Analyses were carried out in both PI and NI modes. Similar sensitivity was obtained for the isomeric flavonoids aglycone in both ionisation modes. Only luteolin was baseline separated (RT $=1.4 \mathrm{~min}$ ), while datiscetin and kaempferol coeluted $(\mathrm{RT}=1.7 \mathrm{~min})$. Those two

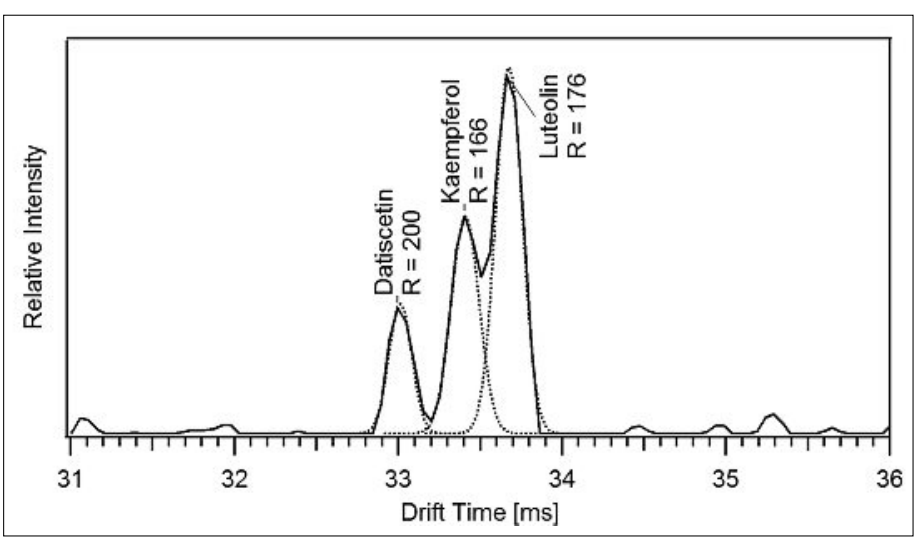

flavonoids are less polar than luteolin in the mobile phase, presumably because of internal hydrogen bonding.

Of the glucosides, the only compound that exhibited a baseline separation was L-6-C (RT $=0.97 \mathrm{~min})$ while coelution of $\mathrm{K}-3-\mathrm{O}, \mathrm{K}-7-\mathrm{O}(\mathrm{RT}=1.20 \mathrm{~min})$ and, partly, of L-7-O (RT = $1.13 \mathrm{~min})$ was observed.

A longer analysis time or an optimized gradient may provide a better separation of the coeluting aglycones and glycoside isomers, but long UHPLC-TOF-MS gradient methods are often not feasible in high-throughput metabolomics studies.

\section{IMS-ESI-MS}

Three different ion mobility methods were tested: two PI modes (with and without the addition of twofold molar excess sodium chloride to the analyte solution); and a single NI mode. The addition of group I metal ion salts is a common approach to improve the separation of carbohydrate isomers and their glyco-conjugates, as cation adduction can stabilize or induce specific gas-phase conformations. ${ }^{[17,18]}$ Other ions could be used and might provide better separations but sodium is often present in natural extracts and was therefore considered the most appropriate and relevant choice.

As might be expected from the multiple potential deprotonation sites of the analytes, best sensitivity was achieved in NI mode. Unfortunately, the deprotonated species were poorly separated by ion mobility. In contrast, the isomeric flavonoids datiscetin, kaempferol and luteolin were satisfactorily resolved in PI mode, as seen in Fig. 3.

Baseline separation was obtained for datiscetin, whereas kaempferol and luteolin partially overlapped. However, the separation of this pair was sufficient for unambiguous peak fitting to determine the relative intensities of these independent features. The observed resolving power for datiscetin is distinctly higher than for the others, $200 v s$. about 170 (FWHM/drift time). The higher value is more representative of the intrinsic instrument perfor-
Fig. 3. Single ion mobility chromatogram of the protonated flavonoids datiscetin, kaempferol and luteolin in PI mode. The indicated resolving powers $(\mathrm{R})$ are based on gaussian peak fits (dashed traces). 
mance, and is greater than the pulsed-mode diffusion limit of about 140.[19] This is an advantage of the post-processing possible with the HT IMS method.

The differences in resolution observed are presumably a reflection of the conformational stabilities of the respective species. Intramolecular hydrogen bonding between the phenolic and chromone hydroxyl groups likely stabilizes datiscetin in a single conformation. In contrast there is no such intramolecular rotational stabilization of the phenol ring in kaempferol and luteolin. By sampling a larger conformational space the IMS peaks become broader, and the drift times slightly longer. The reduced mobilities and collisional cross sections are listed in Table 1.

The flavonoids were not observed to readily form sodium adducts upon addition of sodium chloride to the spray solution. This is in agreement with observations reported by Hill and co-workers ${ }^{[18]}$ and probably due to collisional cooling of the ions during the ion mobility separation, leading to the loss of less favourable transition states prior to mass analysis.

The single ion mobility chromatograms of the glyco-conjugates of kaempferol and luteolin are depicted in Fig. 4. Good signal was observed for all the three experimental modes, the sugar moiety of the glycosides readily complexed the alkali ions in the gas phase. Slightly better separation was achieved in PI compared to NI mode, although the 7-O-glucoside of kaempferol and luteolin appeared as a single peak in all cases (confirmed by measurement of the individual compounds).

Cationisation with sodium did not lead to greatly improved separation of the glycosides, in contrast to initial expectations. Interpeak separation with sodium was perhaps greater than without, but only slightly so. While it is desirable to have the ability to modify the separation with additives, it is also convenient if protonation (or deprotonation) gives good performance. Metal-ion adducts often exhibit lower intensities compared to protonated species and require an additional step in sample preparation. Also, the co-existence of both metal-ion adducts and protonated species leads to complicated spectra for complex samples which require more effort to interpret.

\section{Conclusions}

Rapid UHPLC-MS and IMS-MS methods for analysis of closely related isomeric flavonoids and their glycosides were investigated with a test set of seven species. On an experimental time scale of four minutes, complete separation of all seven was not achieved with either UHPLC or IMS-MS.
Table 1. Reduced mobilities and ion-neutral collision cross sections of the protonated species included in this study, in nitrogen as drift gas. The estimated $\mathrm{K}_{0}$ and cross section uncertainties are $\pm 0.3 \%$.

\begin{tabular}{|l|l|l|l|}
\hline Analyte & M. W. $\mathbf{~ g / m o l ] ~}$ & $\mathbf{K}_{\mathbf{0}}\left[\mathbf{c m}^{2} / \mathbf{V s}\right]$ & $\mathbf{\Omega}\left[\AA^{2}\right]$ \\
\hline Datiscetin & 286.23 & 1.265 & 149.2 \\
\hline Kaempferol & 286.23 & 1.249 & 151.2 \\
\hline Luteolin & 286.23 & 1.240 & 152.0 \\
\hline K-3-O & 448.38 & 0.978 & 189.9 \\
\hline L-6-C & 448.38 & 0.961 & 193.3 \\
\hline K-7-O & 448.38 & 0.939 & 197.8 \\
\hline L-7-O & 448.38 & 0.939 & 197.8 \\
\hline
\end{tabular}

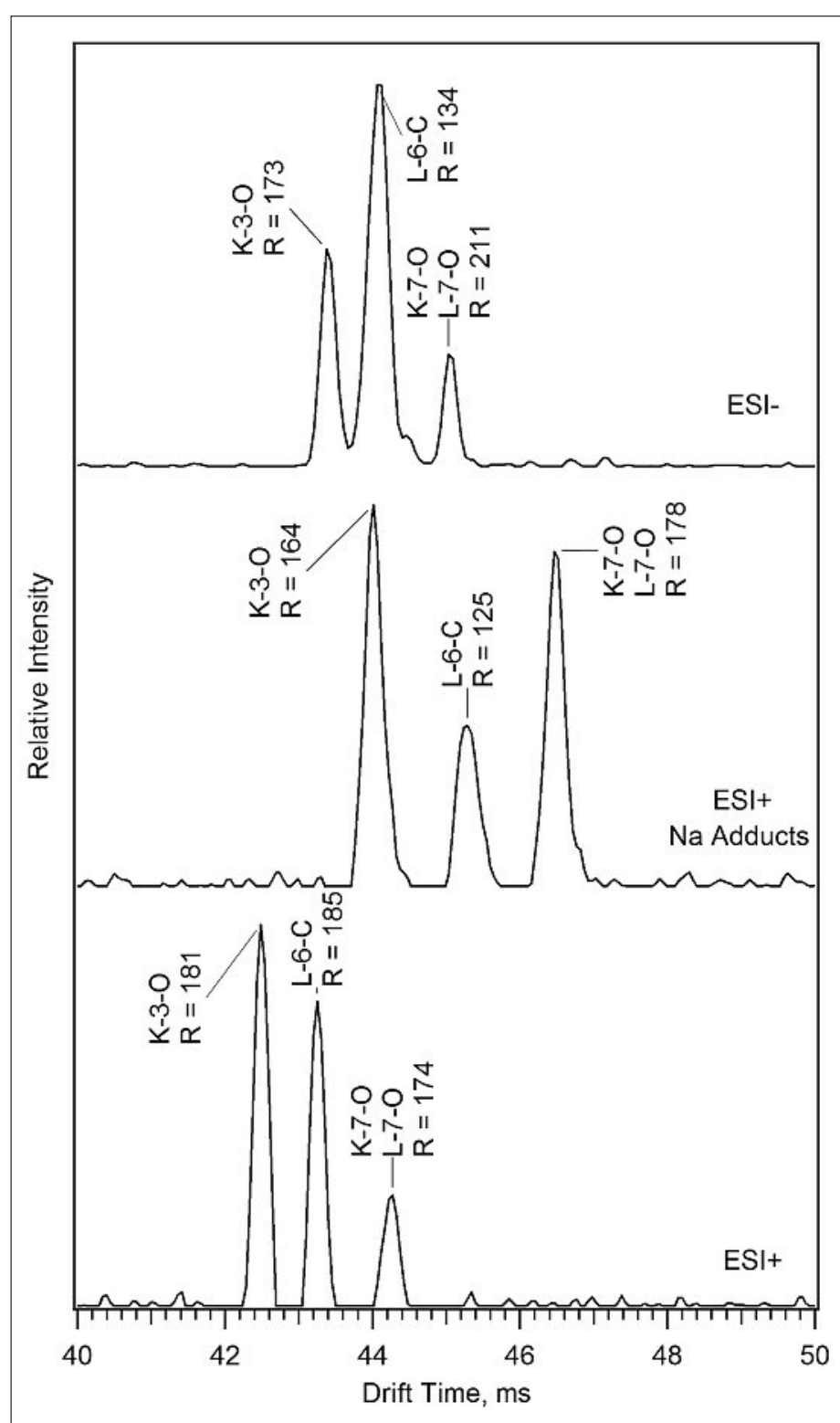

Fig. 4. Separation of flavonoid glycosides in PI mode (bottom; $\left.[\mathrm{M}+\mathrm{H}]^{+}\right)$, PI mode with the addition of sodium chloride (middle; $[\mathrm{M}+\mathrm{Na}]^{+}$) and $\mathrm{NI}$ mode (top; [M-H] $]^{-}$.
This time corresponds to the gradient in UHPLC or the averaging time in IMS-MS.

With UHPLC, the three isomeric flavonoids separate into two peaks. The relative response factors differ slightly in PI and NI modes, but all were detected with similar sensitivity. With IMS-MS, the flavonoids were all separated as protonated adduct ions, but not in deprotonated form.
This illustrates a fundamental difference between LC and IMS. In the former, the solution phase separation is independent of ionization in the mass spectrometer, while in the latter, the same ions are separated and mass analysed.

Datiscetin is well separated from kaempferol and luteolin in IMS-MS, but the latter two partially overlap. This proba- 
bly reflects the lack of rotational hindrance of the phenolic ring in kaempferol and luteolin. Substitution on this ring is the sole difference between them. Rotation of the ring broadens the IMS peaks by averaging out the structural differences.

As expected, the glycosides were somewhat more challenging than the free flavonoids. With UHPLC, three peaks were observed, two of which strongly overlapped. In IMS, three of the four were separated in both polarities (protonated or deprotonated), and also as the sodium adduct ion. The 7-O glycosides of luteolin and kaempferol were not resolved. This is consistent with the limited separation of the corresponding aglycones. Addition of the flexible O-linked sugar increases the conformational lability of the species, preventing resolution by IMS.

Based on the flavonoids tested, both UHPLC-MS and IMS-MS show potential for rapid fingerprinting metabolomics of natural extracts. IMS-MS separations were somewhat better, and IMS has intrinsic advantages in reproducibility and transferability of $\mathrm{K}_{0}$ values. On the other hand, infusion IMS is affected by suppression effects caused by competition for charge in electrospray. Optimal coverage, speed and separation are expected by combining LC and IMS. Such investigations of complex natural extracts are ongoing and will be reported later.

\section{Acknowledgements}

JLW is thankful to SNF (grant 200020_146200) for supporting the development of innovative metabolomics studies. Soura Challal (UNIGE) is acknowledged for the recording of the UHPLC-MS and Davy Guillarme (UNIGE) for the design of the UHPLC experiments.

Received: February 4, 2014

[1] P. J. Eugster, D. Guillarme, S. Rudaz, J.-L. Veuthey, P.-A. Carrupt, J.-L. Wolfender, J. AOAC Int. 2011, 20, 51.

[2] J. L. Wolfender, G. Glauser, J. Boccard, S. Rudaz, Nat. Prod. Commun. 2009, 4, 1417.

[3] 'Flavonoids in health and disease', Eds. C. A Rice-Evans, L. Packer, Marcel Dekker Inc., New York, 1998.

[4] H. H. Hill, W. F. Siems, R. H. St. Louis, D. G. McMinn, Anal. Chem. 1990, 62, 1201A.

[5] A. Kanu, P. Dwivedi, M. Tam, L. Matz, H. H. Hill, J. Mass Spectrom. 2008, 43, 1 .

[6] 'Hadamard Transform Optics', Eds. M. Harwit, N. J. A. Sloan, Academic Press, New York, 1979.
[7] P. J. Treado, M. D. Morris, Anal. Chem. 1989, $61,723 \mathrm{~A}$.

[8] B. H. Clowers, W. F. Siems, H. H. Hill, S. M. Massick, Anal. Chem. 2006, 78, 44

[9] A. W. Szumlas, S. J. Ray, G. M. Hieftje, Anal. Chem. 2006, 78, 4474

[10] R. Knochenmuss, S. Graf, K. Fuhrer, M. Gonin, in 'Proc. 61st ASMS Conference on Mass Spectrometry and Allied Topics', 2013, WP745.

[11] Z. Zhang, R. Knochenmuss, W. F. Siems, W. Liu, S. Graf, H. H. Hill, Anal. Chem. 2013, 86, 1661.

[12] G. Kaur-Atwal, G. O'Connor, A. a. Aksenov, V. Bocos-Bintintan, C. L. Paul Thomas, C. S. Creaser, Int. J. Ion Mobil. Spectrom. 2009, 12,

[13] J. Viidanoja, A. Sysoev, A. Adamov, T. Kotiaho, Rapid Commun. Mass Spectrom. 2005, 19 , 3051.

[14] R. Fernández-Maestre, C. S. Harden, R. G. Ewing, C. L. Crawford, H. H. Hill, Analyst 2010, 135, 1433.

[15] S. Fekete, D. Guillarme, J. Chromatogr. A 2013, 1308, 104.

[16] D. Guillarme, D. T. T. Nguyen, S. Rudaz, J.-L. Veuthey. Eur. J. Pharm. Biopharm. 2008, 68, 430.

[17] Y. Huang, E. Dodds, Anal. Chem. 2013, 85, 9728.

[18] B. H. Clowers, H. H. Hill, J. Mass Spectrom. 2006, 41,339

[19] A. B. Kanu, M. M. Gribb, H. H. Hill, Anal. Chem. 2008, 80, 6610 